\title{
Purulent pericarditis due to Klebsiella pneumoniae pulmonary infection
}

\section{Pericarditis purulenta en infección pulmonar por Klebsiella pneumoniae}

\author{
Gala Caixal Vila*, Eduardo Flores, Guillem Caldentey
}

Servicio de Cardiología, Hospital Clínic Barcelona, Spain

\section{KEYWORDS}

Cardiac tamponade;

Cardiology;

Complications;

Pericardium

\section{PALABRAS CLAVE}

Taponamiento cardiaco;

Cardiología;

Complicaciones;

Pericardio

A 67-year-old man, without medical history, was referred to our center with complaints of chest pain and fever. A chest $x$-ray revealed cardiomegaly, blunt costophrenic angles and bilateral lung condensation (Fig. 1). An echocardiogram showed severe pericardial effusion with signs of tamponade and a rounded image between the liver and the right heart in the subcostal view (Fig. 2). An emergency pericardiocentesis was performed obtaining purulent fluid (Fig. 3) and empirical antibiotic treatment was initiated. A full-body CT scan was performed, showing a hypodense subdiaphragmatic mass, not entirely well located (Fig. 4).

\footnotetext{
* Corresponding author.

E-mail address: caixalgala@gmail.com (G. Caixal Vila).
}

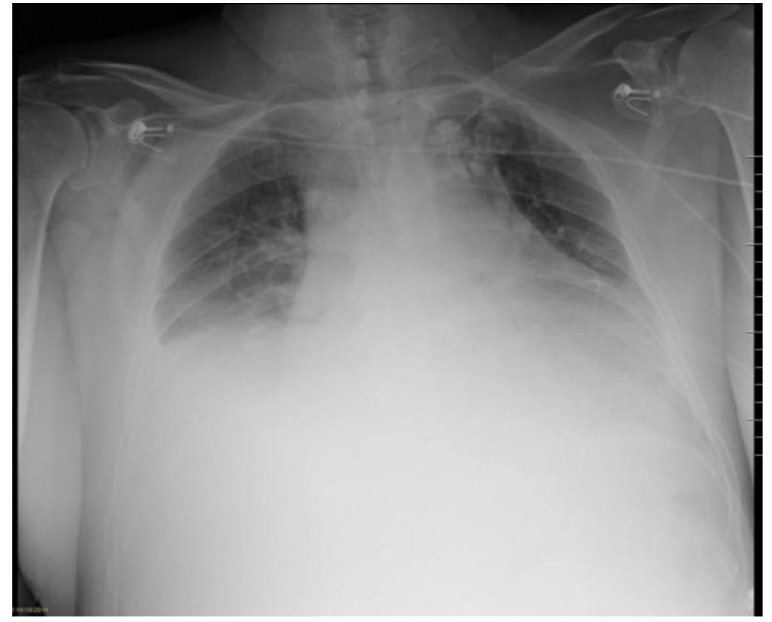

Figure 1

A posterior abdominal ultrasound characterized the finding as a seemingly supradiaphragmatic abscess (Fig. 5), with purulent fluid evidenced by puncture. In both cultures (pericardial liquid and abscess), Klebsiella pneumonia was isolated. Surgical pericardiectomy was carried out and a $7 \times 5 \mathrm{~cm}$ intradiaphragmatic abscess was ultimately located. It was completely drained with good clinical outcomes.

Purulent pericarditis is a rare but life-threatening condition, sometimes with cardiac tamponade as its first manifestation. The primary cause is often non-cardiac, such as extension of an adjacent source of infection or bloodstream dissemination. Classically, most organisms implicated were 


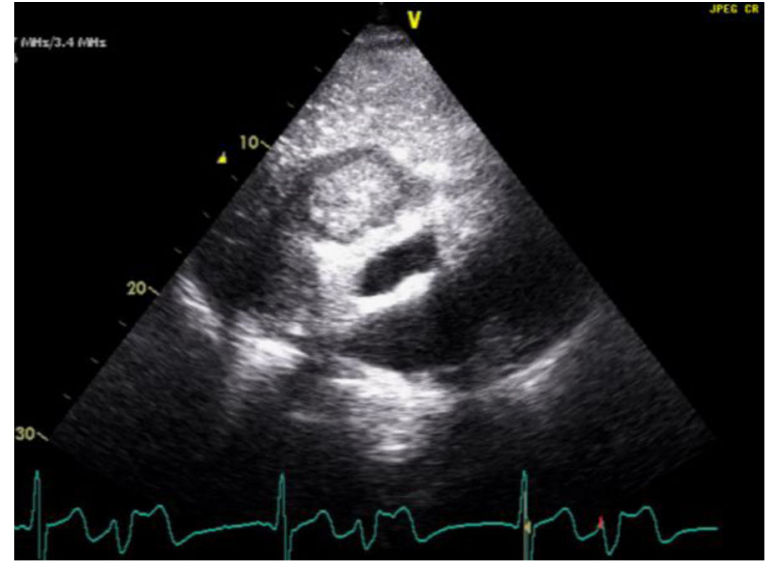

Figure 2

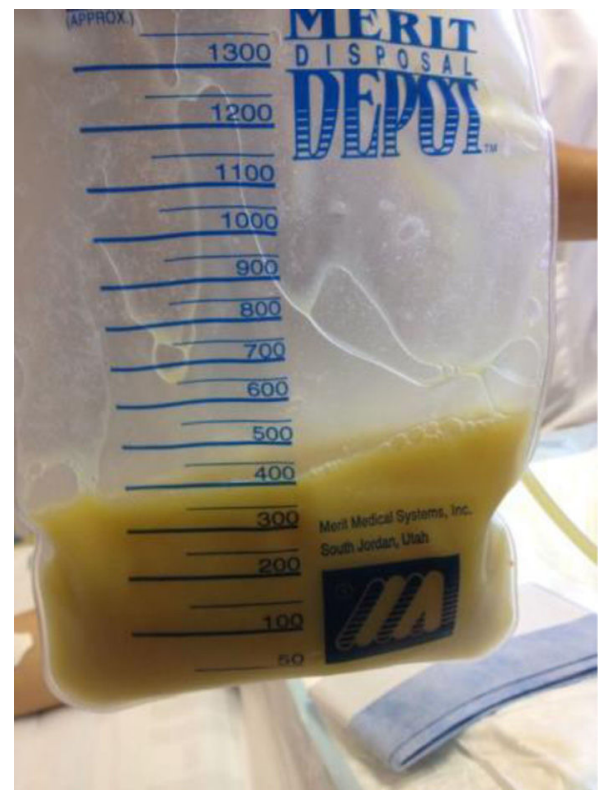

Figure 3

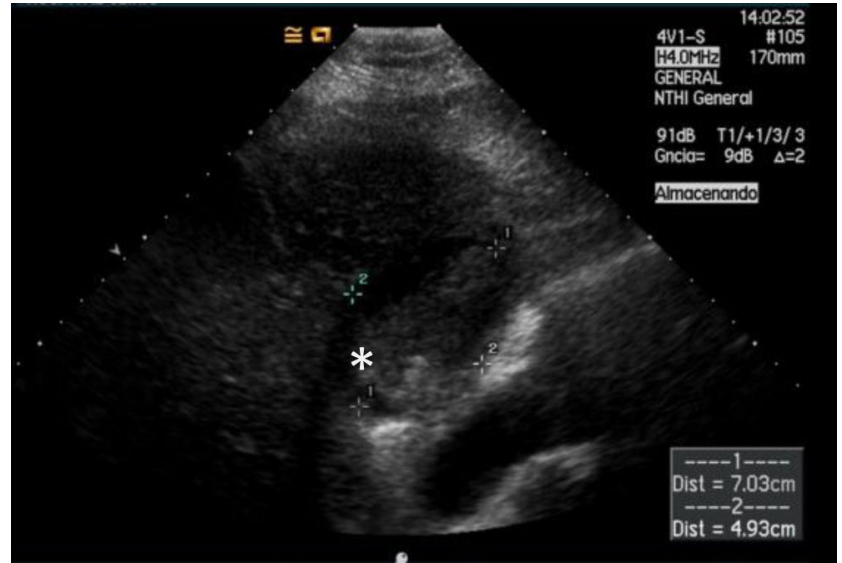

Figure 5

gram positive, predominantly Streptococcus pneumoniae, usually by extension of a pulmonary or abdominal source. Nevertheless, gram negatives, anaerobes and fungi are increasing, reflecting therapeutic advances over the last years, such as antibiotics, chemotherapy, hemodialysis and thoracic surgery. Early diagnosis of pyopericardium and the exclusion of a non-cardiac source of infection, as well as immediate treatment with pericardial tap and intravenous antibiotic therapy may lead to a good outcome.

We report a case of purulent pericarditis with cardiac tamponade due to Klebsiella pneumoniae, an uncommon pathogen barely reported previously.
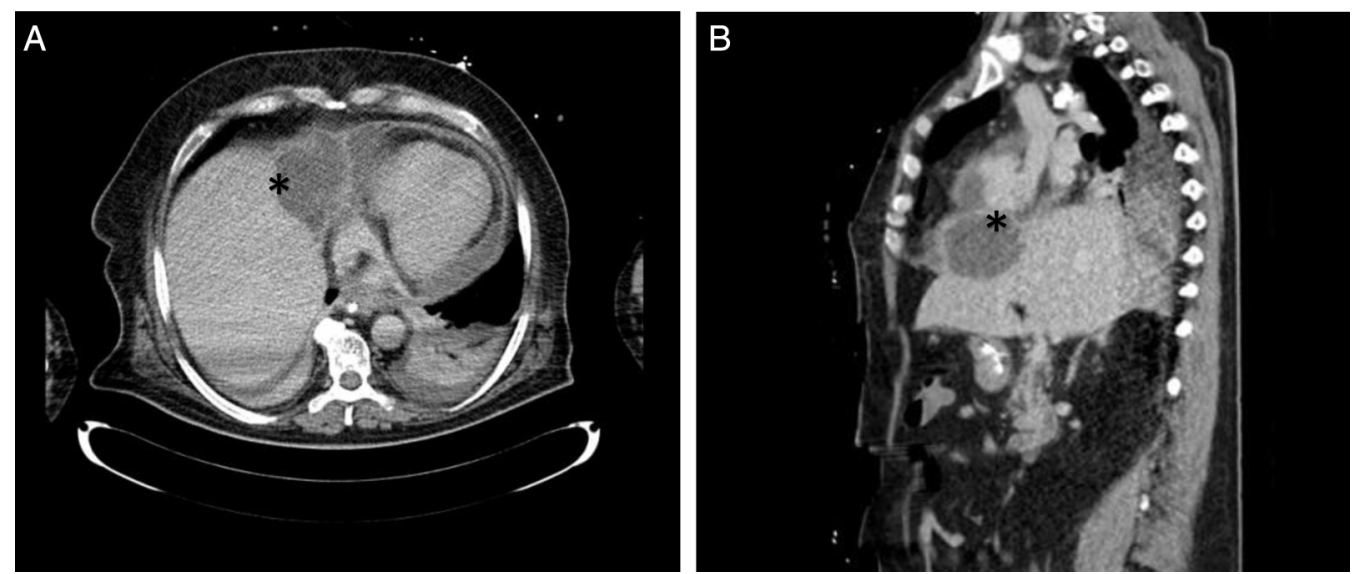

Figure 4 